\title{
Effect of Bone Resection on Posterior Talofibular Ligament Integrity for Posterior Ankle Impingement Syndrome: A Cadaveric Study
}

\author{
Chirapat Inchai, Ph.D., Tanawat Vaseenon, M.D., Yasuhito Tanaka, M.D., and \\ Pasuk Mahakkanukrauh, M.D.
}

\begin{abstract}
Purpose: The purpose of this study was to evaluate the attachment areas of the posterior talofibular ligament (PTFL) on the posterolateral tubercle of the talus and the remaining PTFL attachment areas after consequential bony excision. Methods: Thirty fresh cadaveric ankles were dissected to study the proximal and distal attachment of the PTFL and separated the PTFL into anterior and posterior bundles. The description of the PTFL footprint and the anatomic landmarks from the surrounding structures were analyzed during consequential posterolateral bony excision. Results: The average PTFL dimension was $26.11 \mathrm{~mm}$ (length), $7.65 \mathrm{~mm}$ (width), and $1.82 \mathrm{~mm}$ (thickness). The footprint area of the PTFL on the talar site consists of the posterior bundle $(76.82 \%)$ and the anterior bundle $(23.18 \%)$. If posterolateral tubercle excision was stayed up to a line of a bottom of the flexor hallucis longus (FHL) groove, at least $89 \%$ of the PTFL can be preserved. Conclusion: The posterior bundle of the PTFL is the main bundle on the talar footprint area. To maintain the majority of the attachment of the PTFL, the resection of the posterolateral process could be performed to the bottom of the FHL tendon groove. If resection reaches to the posterior articular cartilage, less than $50 \%$ of the PTFL will be preserved. Understanding the footprint of the PTFL plays a key role in posterior ankle impingement surgery. Clinical Relevance: This study provides guidance for resection of the posterolateral tubercle of the talus and a portion of the PTFL attachment for posterior ankle impingement syndrome. Too much resection of the tubercle may cause instability symptoms.
\end{abstract}

$\mathbf{P}$ osterior ankle impingement syndrome (PAIS) is commonly defined as posterior ankle pain caused by overuse of ankle motion in the plantarflexion movement. It causes increasing stress to the posterior

From the Department of Anatomy, Faculty of Medicine, Chiang Mai University, Chiang Mai (C.I., P.M.); the Department of Orthopedics, Faculty of Medicine, Chiang Mai University, Chiang Mai (T.V.); the Excellence in Osteology Research and Training Center (ORTC), Chiang Mai University, Chiang Mai (P.M), Thailand; and the Department of Orthopaedic Surgery, Nara Medical University, Kashihara, Japan (Y.T.).

The authors report the following potential conflicts of interest or sources of funding: This work was supported by research funding from the Faculty of Medicine, Chiang Mai University, Thailand and Excellence in Osteology Research and Training Center (ORTC), Chiang Mai University, Thailand. Full ICMJE author disclosure forms are available for this article online, as supplementary material.

Received September 27, 2020; accepted January 27, 2021.

Address correspondence to Pasuk Mahakkanukrauh, M.D., Department of Anatomy $\theta$ Excellence in Osteology Research and Training Center (ORTC), Chiang Mai University, 50200, Thailand.E-mail: pasuk034@gmail.com

(C) 2021 THE AUTHORS. Published by Elsevier Inc. on behalf of the Arthroscopy Association of North America. This is an open access article under the CC BY-NC-ND license (http://creativecommons.org/licenses/by-nc-nd/4.0/). 2666-061X/201615

https://doi.org/10.1016/j.asmr.2021.01.025 part of the tibial plafond and the posterior process of the talus. The PAIS is usually found in athletes and ballet dancers. ${ }^{1}$ The posterior part of the talus is an irregular bone surface composed of posterolateral and posteromedial tubercles, and the groove for the flexor halluces longus tendon lies between these 2 tubercles. ${ }^{1,2}$ The pathology of PAIS is varied from bony variation to a fracture of the posterolateral tubercle of the talus. The bone variation of posterolateral tubercle of the talus is the failure of fusion of the secondary ossification center as os trigonum. It presents in $7 \%$ to $14 \%$ of adults, which is bilateral in $1.4 \%$ of cases. Another bony variation is the elongation of the posterolateral tubercle of the talus as the Stieda process. The repetitive stress may cause disruption of the synchondrosis of the os trigonum or chronic fracture of the posterolateral process of the talus. Symptoms of PAIS are pain at the posterior part of the ankle and limited ankle plantarflexion motion during activities. PAIS is caused by impingement of the posterolateral tubercle of the talus and surrounding soft tissues.

The surgical treatment including open and endoscopic techniques is the resection of the bony ossicles either os trigonum or Stieda's process. Adequate bony resection 
could be confirmed intraoperative by passive ankle plantarflexion under fluoroscopy. However, the posterior talofibular ligament (PTFL) is attached posterior part of the talus. There were no adequate data how much resection causes how much PTFL is scarified. The PTFL is a thick bundle and a strongest ligament of posterior ankle ligament complex. ${ }^{3}$ The proximal attachment of PTFL locates on the fossa of medial surface of the fibula. The bundle of ligament runs posteromedially and horizontally from the proximal attachment site in the malleolar fossa of fibula to distal attachment on the posterolateral aspect of the talus. Rasmussen et al. ${ }^{4}$ revealed that the role of PTFL, anterior and posterior bundles has no independent role in maintaining stability of the joint when the anterior talofibular ligament (ATFL) and calcaneofibular ligament (CFL) are intact. After the rupture of ATFL and CFL, the anterior fiber of PTFL restrict internal and external rotation, talar tilt and dorsiflexion, while the posterior fiber of PTFL inhibit only external rotation, talar tilt and dorsiflexion. ${ }^{4}$

On the recent studies of the anatomic footprint of the ankle ligaments, numerous studies reported the gross anatomy of the ATFL, the CFL, syndesmotic ligament such as anterior tibiofibular ligament and PTFL to assess the morphology, origins, insertions, number of the bands, and location of the footprint of each ligament. ${ }^{5-8}$ Previous studies have reported on the anatomic variation of the lateral ligament complex of the ankle joint, including the shape, size, length, and direction, with various methods such as 3-dimensional computed tomography imaging, ${ }^{9}$ magnetic resonance imaging (MRI), ultrasonography, and cadaveric gross dissection-fresh frozen and formalin fixation cadavers. ${ }^{6}$ The knowledge of the footprint anatomy and variation is the key of success for endoscopic and reconstructive surgeries. However, the footprint or morphology studies of the PTFL are rare. The exact and clear understanding in the anatomy of the PTFL is essential to the diagnosis and treatment of the posterior ankle surgery. When performing the hindfoot surgery, the surgeon has little guidance on the location of the exact attachment area of the PTFL.

The purpose of this study was to evaluate the attachment areas of the PTFL on the posterolateral tubercle of the talus and the remaining PTFL attachment areas after consequential bony excision. We hypothesized that resection of the posterior process to a point at the bottom of the flexor hallucis longus (FHL) groove was adequate for preserving PTFL.

\section{Methods}

A total of 30 legs of fresh frozen cadavers; 4 females and 11 males were obtained. The age at death ranged from 20 to 80 years. No history of lower-extremity injury, pathology, or ankle deformity was reported.
Ethical approval for the present study was obtained from the ethics committee from the Faculty of Medicine, Chiang Mai University. Soft tissue around the ankle was dissected. The PTFL and its attachments on the fibula and talus were preserved. The morphologic study of the PTFL was recorded by sequential dissection. A quantitative descriptive morphologic study was conducted. There were 2 bundles of PTFL: the anterior bundle that had the footprint more anterior on the fibular attachment and the posterior bundle that had the footprint posterior to the first bundle. On the talar attachment, the posterior bundle consisted of 2 uppermost bands. The anterior bundle was the lower band. The length of each bundle and the footprints was calculated. The attachment sizes of the ligaments were evaluated in 2 areas: fibular and talar attachments. The technique of attachment area calculation was precisely by separation dissection into small multiple bands. Each band was retracted, and each footprint was marked. The acrylic colors were used as a marker point of specific attachment point of ligament bundles and photographed with a camera.

With the purpose of bony resection with less compromising of the PTFL, authors used anatomic landmarks from the surrounding structures of the posterolateral tubercle of the talus including the groove of the FHL tendon and the articular surface of the talus. The posterolateral tubercle of the talus was divided into 4 levels. An imaginary line that runs perpendicular to the axis of the talar articular surface was drawn at the tip of the posterolateral tubercle. The secondary line was drawn at the groove of the FHL tendon. The first line bisected the imaginary line and the secondary line. The fourth line was parallel to the second line at the articular surface of the talus. The third line bisected the second and fourth lines. The photographs were made in superior and posterior views for data collection. The sizes of the footprints in each level were measured with Image $\mathrm{J}$ software. All statistical analysis tests were performed with SPSS version 17. $P<.05$ was considered to be significant.

\section{Results}

The PTFL was composed of 2 bundles. The posterior bundle was the long bundle that ran downward and horizontally from the fibular site and attached to the posteromedial and posterolateral tubercles of the talus. The anterior bundle was the short bundle that ran horizontally and was attached to the posterolateral tubercle of the talus (Fig 1). The average length of the PTFL was $26.11 \pm 3.31 \mathrm{~mm}$. The average width of the PTFL on the fibular site, middle, and talar site was $6.98 \pm 1.39$ $\mathrm{mm}, 7.65 \pm 1.15 \mathrm{~mm}$, and $9.00 \pm 1.45 \mathrm{~mm}$, respectively. Moreover, the average thickness of the PTFL on the fibular, middle, and talar site was $2.01 \pm 0.64 \mathrm{~mm}$, $1.81 \pm 0.51 \mathrm{~mm}$, and $0.44 \pm 0.55 \mathrm{~mm}$, respectively. 


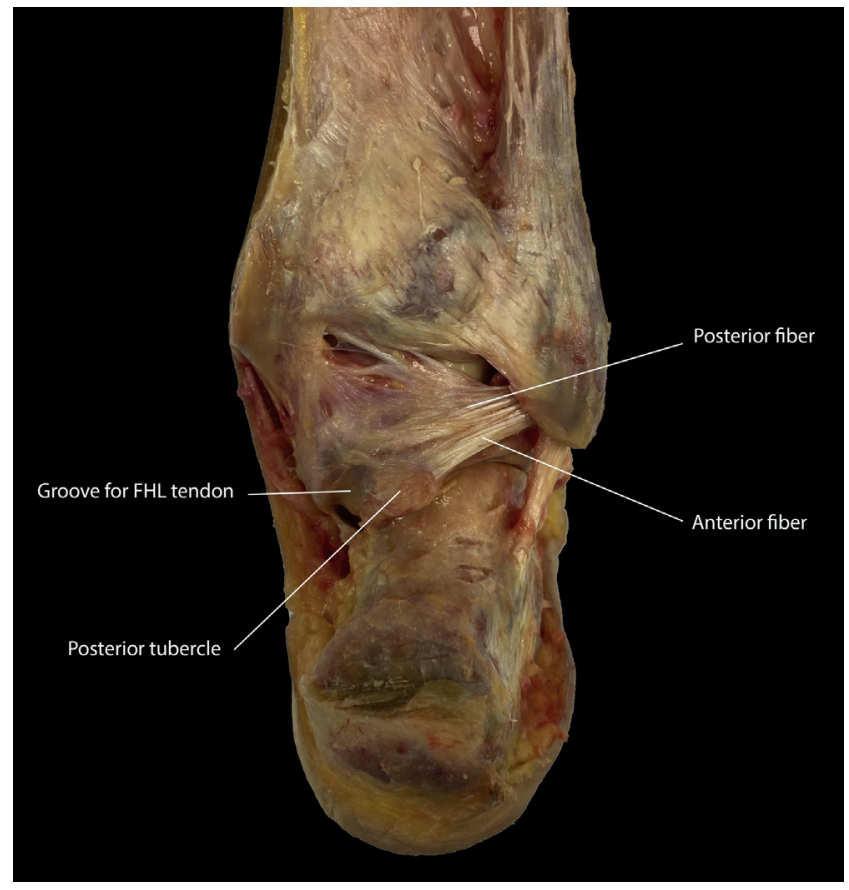

Fig 1. The posterior ankle ligament.

The average distance from the tip of the posterolateral tubercle of the talus to the PTFL ligament was $4.36 \pm$ $1.53 \mathrm{~mm}$, with a minimum of $1.83 \mathrm{~mm}$ and maximum of $7.15 \mathrm{~mm}$. The average area between the tip of the posterolateral tubercle of the talus to the PTFL ligament was $52.80 \pm 18.75 \mathrm{~mm}^{2}$ with a minimum of $18.10 \mathrm{~mm}^{2}$ and a maximum of $85.63 \mathrm{~mm}^{2}$.

The footprint area of the PTFL on the fibular site was $74.04 \pm 15.68 \mathrm{~mm}$, with a minimum of $47.75 \mathrm{~mm}$ and a maximum of $111.83 \mathrm{~mm}$. The footprint areas of the PTFL on the talar site (Fig 2) were described in total, anterior bundle, and posterior bundle in Table 1 . In addition, with 4 levels of bony landmark excision (Figs 3 and 4), the footprint areas of the PTFL on the talus were described in superior and posterior views in Table 2.

\section{Discussion}

The footprint area of the PTFL on the talar site consists of approximately $77 \%$ posterior bundle and $23 \%$ anterior bundle. To maintain $89 \%$ of the attachment of the posterior bundle, the resection of the posterolateral process could be performed up to the bottom of the FHL tendon groove. If the resection is made to the cartilage, $41 \%$ of the PTFL would be preserved. PAIS may result from various factors such as anomalous muscle, soft tissue or bony impingement, scar tissue impingement in the posterior part of the ankle joint, or the variation of bony structures includes the elongation of the posterior process of the talus also known as the Stieda's process and the os trigonum, an accessory bone which is the failure fusion of the secondary ossification. Soft tissue impingement is caused by an accessory ligament, such as the posterior intermalleolar ligament, the posterior inferior tibiofibular ligament, or the PTFL. ${ }^{2}$ The ligament may protrude further into the joint during ankle plantarflexion, becoming entrapped and torn. The bony structures responsible for PAIS include the posterior subtalar joint, the posterolateral process of the talus, and the os trigonum. Tokgöz et al. ${ }^{10}$ investigated the relationship between the pathologies of the flexor hallucis longus tendon, including injury to the FHL tendon, degeneration, and tenosynovitis related to the presence of the os trigonum. The result showed that the extent of the os trigonum can increase the lesions to the FHL tendon. For bone and ossicle lesions, failure of fusion between the secondary ossification center and the posterolateral tubercle of the talus is a result of the ossicle called the os trigonum, which is the partially attached area of the PTFL. ${ }^{11}$ Another pathology is the elongation of the posterolateral process of the talus, also called Stieda's process, which is also the attachment

Fig 2. Specific attachment areas of the posterior talofibular ligament (a) on the superior and (b) posterior views. The posterior bundle footprint is in green and yellow. The anterior bundle footprint is in red.
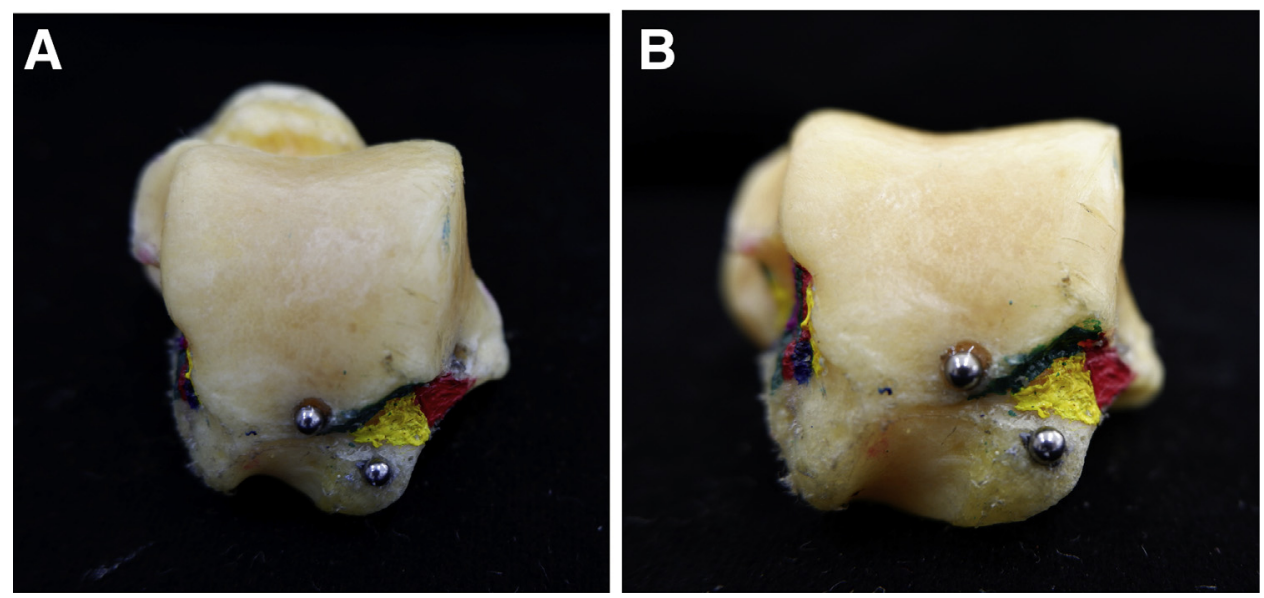
Table 1. Descriptive Analysis of the Footprint Area of the PTFL on the Talar Site (Average, Standard Deviation, Minimum and Maximum).

\begin{tabular}{|c|c|c|c|c|}
\hline Total Area $\left(\mathrm{mm}^{2}\right)$ & Anterior Bundle Area $\left(\mathrm{mm}^{2}\right)$ & Percentage & Posterior Bundle Area $\left(\mathrm{mm}^{2}\right)$ & Percentage \\
\hline $81.61 \pm 28.93(33.80-147.47)$ & $18.90 \pm 9.73(4.79-38.07)$ & $23.18 \%$ & $62.71 \pm 23.20(25.05-109.41)$ & $76.82 \%$ \\
\hline
\end{tabular}

area of the PTFL. In the literature, a few studies provide exact and clear anatomic information about the PTFL, which attaches to the os trigonum or the posterolateral process of the talus. Gursoy et al. ${ }^{12}$ studied the details of PTFL on MRI in patients with os trigonum. They found that when os trigonum appeared, the PTFL was inserted within the os trigonum.

Both the open and endoscopic bony excision yield acceptable clinical outcomes in terms of function and pain at 5 years' follow-up. However, endoscopy has advantages in minimized soft tissue injury, fewer complications, and early return to full activities. ${ }^{13}$ The 2-portal hindfoot endoscopic bony excision for the PAIS showed excellent results and decreasing postoperative complications. ${ }^{14}$ With the limitation of visualization compared to open excision, understanding anatomic variations of the ankle could reduce the risk of iatrogenic injuries during ankle surgical procedures. ${ }^{15}$ From a surgical point of view, it is very important to know the precise anatomic information and the variations, which are beneficial for experienced and inexperienced surgeons. In the present study we aimed to establish the specific anatomic description of the attachment area or footprint of each bundle of PTFL, especially in the talar attachment site, and to apply to the operation of bony excision for treating PAIS and to improve the knowledge of the exact anatomic information of PTFL to decrease the complications of over-detachment of PTFL, which may cause chronic ankle instability.

In 2008, Courvoisier et al. ${ }^{16}$ conducted an anatomic study that the PTFL was divided into 2 bundles: the anterior short bundles that attach from the medial site of the lateral malleolus to the posterior edge of the talus and the posterior long bundle that attach to the posterior process of the talus at the talar site. It is also confirmed by the MRI study of Gursoy and colleagues
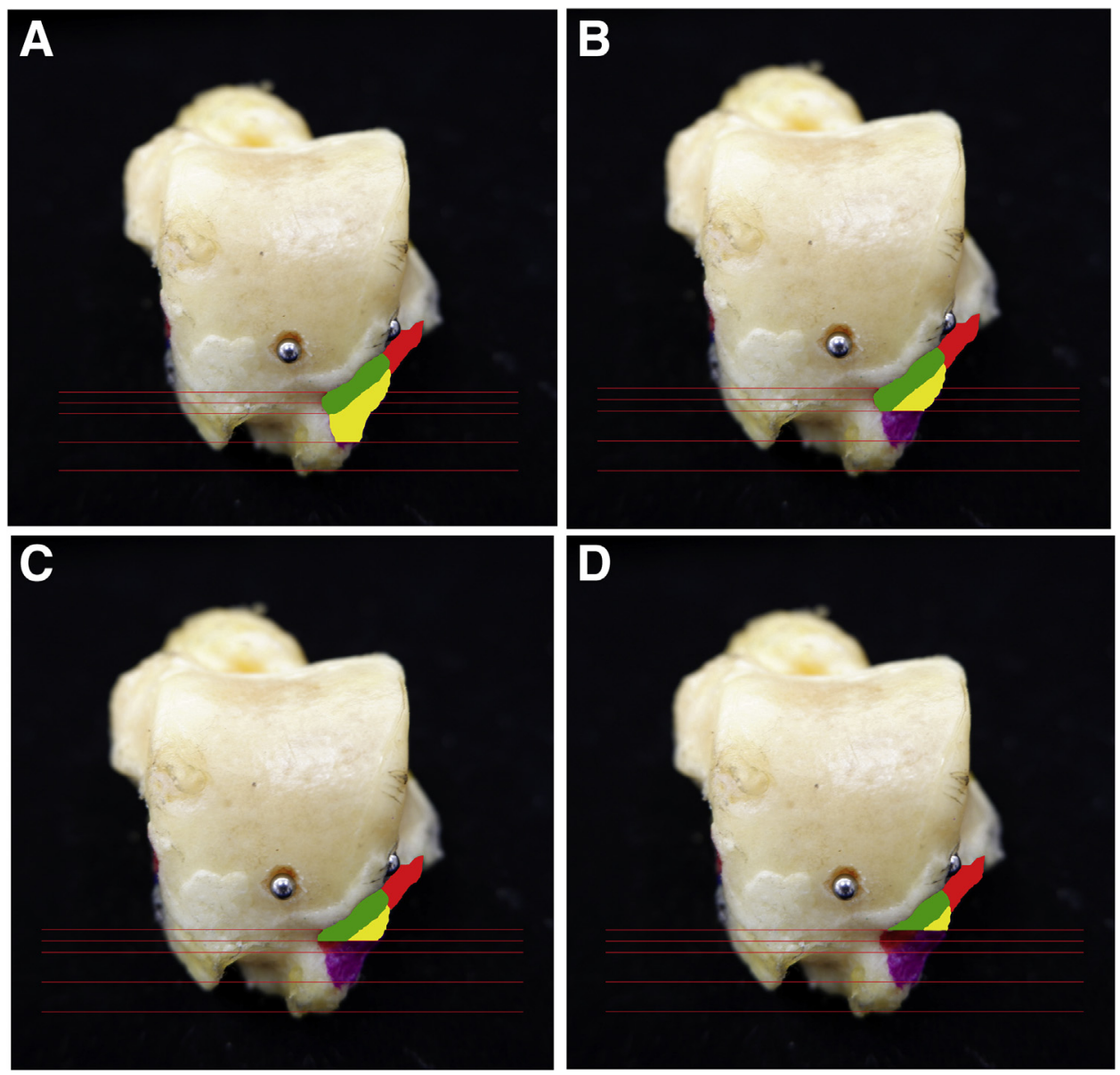

Fig 3. The 4 levels of the bony excision and the remaining area at the superior views of the posterior talus site; (a-d) defined as lines 1-4 of bony excision, respectively. The posterior bundle footprint is in green and yellow. The anterior bundle footprint is in red. 
Fig 4. The 4 levels of the bony excision and the remaining area at the posterior views of the posterior talus site; (a-d) defined as lines 1-4 of bony excision, respectively. The posterior bundle footprint is in green and yellow. The anterior bundle footprint was in red.
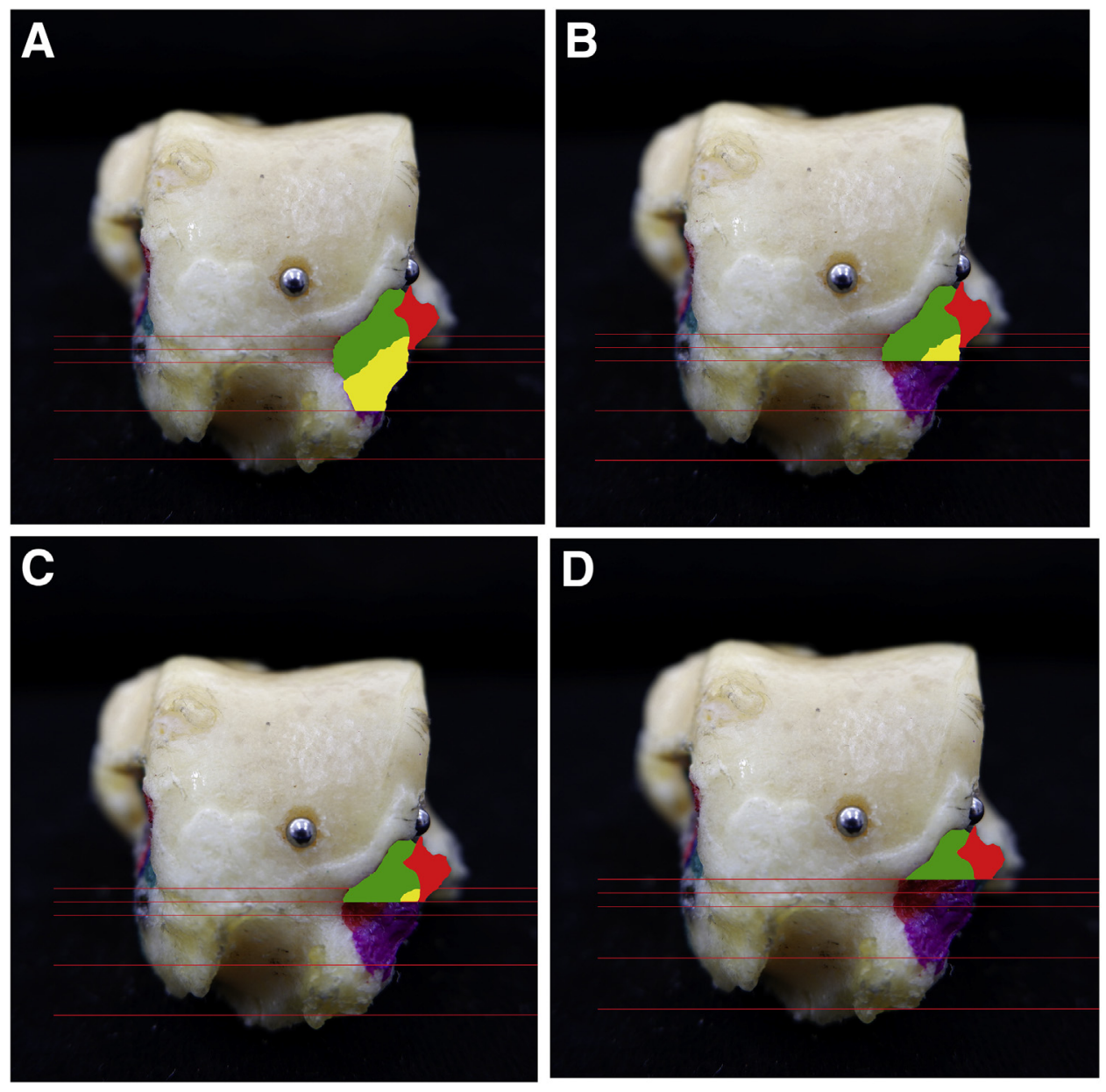

in 2015. ${ }^{12}$ Among the previous studies of PTFL, both MRI and cadaveric dissection obtained similar results and described the PTFL bundles as anterior and posterior bundles in the anatomic literature. The anatomy and function of each bundle may be a role of the posterolateral ankle stability. As a limitation of anatomic cadaveric studies, there is a limited number of published studies that mention the footprint of the PTFL in the Asian population, whose anatomic footprints of ligaments may be smaller than those of
Caucasians. Basic knowledge of the size of the ligament footprint and the presence of exact bony landmarks in the Asian population will be a benefit for orthopaedic surgeons to understanding the anatomy of bone excised and the involvement of the PTFL attachment.

The size of the attachment area of the PTFL at the talus site was highly variable, as was reported in previous studies. Despite this variability, Wenny et al. ${ }^{17}$ in 2014 investigated the length and width dimensions of all lateral ankle ligaments in comparison to previous

Table 2. Descriptive Analysis of the Footprint Area of the PTFL at the Talar Site in Different Level of Bony Excision.

\begin{tabular}{|c|c|c|c|c|c|c|}
\hline Bony Excision & $\begin{array}{c}\text { Average of Total } \\
\text { Remaining Area }\left(\mathrm{mm}^{2}\right)\end{array}$ & Percentage & $\begin{array}{c}\text { Remaining Area of } \\
\text { Anterior Bundle }\left(\mathrm{mm}^{2}\right)\end{array}$ & Percentage & $\begin{array}{c}\text { Remaining Area of } \\
\text { Posterior Bundle }\left(\mathrm{mm}^{2}\right)\end{array}$ & Percentage \\
\hline \multicolumn{7}{|l|}{ Superior view } \\
\hline Level 1 & $62.57 \pm 21.31$ & $99.18 \%$ & $16.88 \pm 7.94$ & $100 \%$ & $45.70 \pm 18.51$ & $98.89 \%$ \\
\hline Level 2 & $55.48 \pm 16.33$ & $89.93 \%$ & $16.58 \pm 7.56$ & $98.99 \%$ & $38.90 \pm 13.84$ & $87.42 \%$ \\
\hline Level 3 & $45.95 \pm 12.23$ & $75.71 \%$ & $16.13 \pm 7.15$ & $97.21 \%$ & $29.82 \pm 9.71$ & $69.06 \%$ \\
\hline Level 4 & $32.78 \pm 9.55$ & $54.90 \%$ & $15.24 \pm 6.40$ & $93.47 \%$ & $17.54 \pm 7.20$ & $42.35 \%$ \\
\hline \multicolumn{7}{|l|}{ Posterior view } \\
\hline Level 1 & $81.06 \pm 28.47$ & $99.48 \%$ & $18.90 \pm 9.73$ & $99.98 \%$ & $62.16 \pm 22.87$ & $99.30 \%$ \\
\hline Level 2 & $71.28 \pm 24.06$ & $88.84 \%$ & $17.91 \pm 9.43$ & $95.36 \%$ & $53.36 \pm 18.91$ & $87.31 \%$ \\
\hline Level 3 & $53.53 \pm 13.76$ & $69.15 \%$ & $14.93 \pm 7.36$ & $83.55 \%$ & $38.60 \pm 10.99$ & $66.08 \%$ \\
\hline Level 4 & $30.67 \pm 10.87$ & $41.25 \%$ & $9.87 \pm 5.56$ & $59.87 \%$ & $20.80 \pm 8.32$ & $37.43 \%$ \\
\hline
\end{tabular}


studies. Seventeen of the formalin-fixed cadaveric feet were revealed, and all of the ankle ligaments, including the PTFL, were investigated to provide precise anatomic information. The results showed that the attachment area of the PTFL at the talar site was posterior to the border of the lateral malleolar surface and the trochlear of the talus. Courvoisier et al. ${ }^{16}$ reported that the PTFL attachment on the talar site is trapezoid-shaped. The area of the attachment at the fibular site was $48 \pm 6$ $\mathrm{mm}^{2}$, which was smaller than that in the present study, which was $74.04 \mathrm{~mm}^{2}$. Moreover, the area of attachment at the talar site was $65 \pm 11 \mathrm{~mm}^{2}$; when compared to the present study, the talar attachment site of the PTFL was bigger, with an average area of $100.31 \mathrm{~mm}^{2}$, which might be due to precise dissection and the technique of dividing into multiple bands to identify the footprint. Neuschwander et al. ${ }^{6}$ performed a detailed study of the anatomy of the natural human lateral ankle ligament by dividing it into small bundles for better understanding and clear anatomic ligament reconstruction by using 3-dimensional computed tomography imaging, which is the new method to study the anatomy of the lateral collateral ligament of the ankle joint. The study of footprint of each ligament was performed with radio-opaque paint and computer software used to identify the attachment area of the ligament, as well as to measure the CFL footprint. It is suggested that there is likely no difference in accuracy between the 2 techniques for studying the footprint of the ligament by hand before computed tomography imaging. ${ }^{6}$

Concern about too much dissection of the PTFL attachment during the bony excision procedure is clinically important. We found that the posterior bundle of the PTFL is the majority of the total footprint, whereas the anterior bundle of the PTFL was involved in $23 \%$ of the total footprint, especially in the anterior. In addition, from the present study, we found that only $9.6 \%$ of the cadavers had the PTFL ligament attached less than $2.5 \mathrm{~mm}$ from the tip of the posterolateral process. The average distance from the tip to the posterolateral process of the talus to the PTFL that had no ligament attached was $4.36 \mathrm{~mm}$. We could summarize that it is safe to perform bony excision within 4 $\mathrm{mm}$ from the tip of the posterolateral process without concern about ligament damage.

For posterior ankle surgery (bony excision), the present study found that removing the posterolateral process of the talus to the FHL groove (level II) caused one-fourth removal of the posterior bundle of the PTFL. Moreover, less than $50 \%$ of the posterior bundle of the PTFL remained when removing the posterolateral process of the talus to the talar articular surface (level IV). More than $50 \%$ of the anterior bundle of the PTFL remained even when removing the posterolateral process of the talus to the talar articular surface (level IV). The recommendations are to remove the posterolateral tubercle of the talus until there is no sign of posterior impingement from intraoperative passive plantarflexion test and, if extensive bony excision is needed, the excision should not beyond to the FHL groove (level II).

\section{Limitations}

There were some limitations to our study. First, the cadavers had no previous injury or deformities around the ankle. Second, we did not know important details such as a history of ankle or posterior ankle pain. One specimen may have had Steida's process as the pathology. It may have had some variation of the PTFL attachment in the pathologic case and changed the result and percentage of the footprint in each level of bony excision. Anatomic variations might have influenced the results. Third, there was no biomechanical study supporting the stability after the PTFL was resected.

\section{Conclusion}

The posterior bundle of the PTFL is the main bundle on the talar footprint area. To maintain the majority of the attachment of the PTFL, resection of the posterolateral process could be performed to the bottom of the FHL tendon groove. If resection reaches to the posterior articular cartilage, less than $50 \%$ of the PTFL will be preserved.

\section{Acknowledgment}

The authors thank those who donated the body for study and research.

\section{References}

1. Hamilton WG, Geppert MJ, Thompson FM. Pain in the posterior aspect of the ankle in dancers. Differential diagnosis and operative treatment. J Bone Joint Surg Am 1996;78:1491-1500.

2. Russell JA, Kruse DW, Koutedakis Y, McEwan IM, Wyon MA. Pathoanatomy of posterior ankle impingement in ballet dancers. Clin Anat 2010;23:613-621.

3. Jerosch J, Fadel M. Endoscopic resection of a symptomatic os trigonum. Knee Surg Sports Traumatol Arthrosc 2006;14: 1188-1193.

4. Rasmussen O, Jensen IT, Hedeboe J. An analysis of the function of the posterior talofibular ligament. Int Orthop 1983;7:41-48.

5. Bartoníček J. Anatomy of the tibiofibular syndesmosis and its clinical relevance. Surg Radiol Anat 2003;25:379-386.

6. Neuschwander TB, Indresano AA, Hughes TH, Smith BW. Footprint of the lateral ligament complex of the ankle. Foot Ankle Int 2013;34(4):582-586.

7. Matsui K, Takao M, Tochigi Y, Ozeki S, Glazebrook M. Anatomy of anterior talofibular ligament and calcaneofibular ligament for minimally invasive surgery: A systematic review. Knee Surg Sports Traumatol Arthrosc 2017;25:1892-1902.

8. Matsui K, Oliva XM, Takao M, Pereira BS, Gomes TM, Lozano JM, et al. Bony landmarks available for minimally 
invasive lateral ankle stabilization surgery: a cadaveric anatomical study. Knee Surg Sports Traumatol Arthrosc 2017;25(6):1916-1924.

9. Golanó P, Vega J, de Leeuw PAJ, et al. Anatomy of the ankle ligaments: A pictorial essay. Knee Surg Sports Traumatol Arthrosc 2010;18:557-569.

10. Tokgöz MA, Ataoğlu MB, Ergişi Y, Bozkurt HH, Kanatli U. Is there any effect of presence and size of os trigonum on flexor hallucis longus tendon lesions? Foot Ankle Surg 2020;26: 469-472.

11. Yilmaz C, Eskandari MM. Arthroscopic excision of the talar Stieda's process. Arthroscopy 2006;22:225.el-225.e3.

12. Gursoy M, Dag F, Mete BD, Bulut T, Uluc ME. The anatomic variations of the posterior talofibular ligament associated with os trigonum and pathologies of related structures. Surg Radiol Anat 2015;37:955-962.

13. Georgiannos D, Bisbinas I. Endoscopic versus open excision of os trigonum for the treatment of posterior ankle impingement syndrome in an athletic population: A randomized controlled study with 5-year follow-up. Am J Sports Med 2017;45:1388-1394.

14. van Dijk CN, Scholten PE, Krips R. A 2-portal endoscopic approach for diagnosis and treatment of posterior ankle pathology. Arthroscopy 2000;16: $871-876$.

15. Nayak VS, Bhat N, Nayak SS, Sumalatha S. Anatomical variations in the cutaneous innervation on the dorsum of the foot. Anat Cell Biol 2019;52:34-37.

16. Courvoisier A, Vialle R, thevenin-lemoine C, Mary P, Damsin J-P. The posterior talofibular ligament: An anatomical study with clinical implication in clubfoot surgery. Surg Radiol Anat 2008;30:633-637.

17. Wenny R, Duscher D, Meytap E, Weninger P, Hirtler L. Dimensions and attachments of the ankle ligaments: evaluation for ligament reconstruction. Anat Sci Int 2015;90:161-171. 